\title{
Recent advances on Failure and Recovery in Networks of Networks
}

\author{
Louis M. Shekhtman ${ }^{\mathrm{a}, 1, *}$, Michael M. Danziger ${ }^{\mathrm{a}}$, Shlomo Havlin $^{\mathrm{a}}$ \\ ${ }^{a}$ Department of Physics, Ramat Gan, Israel
}

\begin{abstract}
Until recently, network science has focused on the properties of single isolated networks that do not interact or depend on other networks. However it has now been recognized that many realnetworks, such as power grids, transportation systems, and communication infrastructures interact and depend on other networks. Here we will present a review of the framework developed in recent years for studying the vulnerability and recovery of networks composed of interdependent networks. In interdependent networks, when nodes in one network fail, they cause dependent nodes in other networks to also fail. This is also the case when some nodes, like for example certain people, play a role in two networks, i.e. in a multiplex. Dependency relations may act recursively and can lead to cascades of failures concluding in sudden fragmentation of the system. We review the analytical solutions for the critical threshold and the giant component of a network of $n$ interdependent networks. The general theory and behavior of interdependent networks has many novel features that are not present in classical network theory. Interdependent networks embedded in space are significantly more vulnerable compared to non-embedded networks. In particular, small localized attacks may lead to cascading failures and catastrophic consequences. Finally, when recovery of components is possible, global spontaneous recovery of the networks and hysteresis phenomena occur. The theory developed for this process points to an optimal repairing strategy for a network of networks. Understanding realistic effects present in networks of networks is required in order to move towards determining system vulnerability.
\end{abstract}

\footnotetext{
* Corresponding author

Email address: 1sheks@gmail.com (Louis M. Shekhtman)
} 
Keywords: Complex matter and networks

PACS: $30.03,30.04$

\section{Introduction}

Classical graph studies involved simple random graphs (Erdős-Rényi networks) or regular lattices, however once more data became available about real-world complex systems, researchers quickly discovered that real networks have far more complex structures. First of all, many real networks have some nodes that act as hubs with far more connections than other nodes $[1,2,3]$. Beyond this, many studies have found other non-random structures such as the small-world structure [4], community structure [5, 6], clustering [4], degree-degree correlations [7, 8], and unique spatial structures [9] in networks. Understanding the topological structure of real-world networks has provided insights into fields as diverse as epidemiology [10, 11, 12, 13, 14], climate [15, 16], economics [17, 18], sociology [19], infrastructure [20], traffic [21], physiological networks [22], and brain networks $[23,24]$.

One of the most important properties of networks is their robustness to failures or in other words what fraction of nodes remain connected after some other subset of nodes is removed? To answer this question it is often useful to use percolation theory which tells us what fraction of nodes are in the largest component, $P_{\infty}[25,26]$. Defining functionality based on the size of the largest component makes sense in many contexts. For example, in the case of a communications network it is usually pertinent to ask what fraction of nodes are able to communicate? If $P_{\infty} \approx 1$ then the network is functional and most nodes can easily communicate. However if $P_{\infty} \approx 0$ then very few nodes can communicate and the network is essentially non-functional. The term giant connected component is used when $P_{\infty}$ is a non-zero fraction of an infinite system.

The formal framework of percolation theory in the context of networks involves varying $1-p$, the fraction of nodes removed at random and calculating the corresponding size of the largest component, $P_{\infty}(p)$. In general, for single isolated networks $P_{\infty}(p)$ undergoes a second-order, con- 
tinuous phase transition [27] as $p$ decreases. The point where the transition occurs is typically referred to as $p_{c}$. For $p>p_{c}$ we have $P_{\infty}(p)>0$, but for $p<p_{c}, P_{\infty}(p)=0$. For Erdős-Rényi networks it was found that $p_{c}=1 /<k>$ where $<k>$ is the mean degree of the network $[28,29,30]$. In contrast, for scale-free networks where the degree distribution follows $p(k) \sim k^{-\lambda}$, it was found that for $\lambda<3, p_{c}=0$ [31], indicating that only when essentially all nodes are removed does the giant component reach zero.

Most real networks do not operate in isolation, but are instead merely one system in a network of networks [32, 33, 34, 35, 36, 37, 38]. One type of important relationship between networks is interdependence $[39,40]$. This occurs in infrastructure where power grids may depend on communications systems and in many biological systems where functionality requires numerous organs and metabolic pathways to work together. Another example occurs in sociology where an individual may participate in multiple social networks $[41,42,43]$. We show an example of the complexity of interdependence in modern infrastructure in Fig. 1. Here we will review some of our recent results on interdependent networks and point readers to other articles where they can learn more about the subject.

In interdependent networks there are two types of links, the usual connectivity links that are also present in single networks, as well as a new type of links called dependency links [39, 40, 45, 46, 47, 48, 49]. These dependency links imply that the node at one end of the link relies on the node at the other end of the link to function. Thus if the node on one end of a dependency link fails, then the node on the other end will also fail.

The structure of the network of networks is based on networks having dependency links between them. Possible structures are shown in Fig. 2 and include treelike structures, a loop, and a random-regular configuration where each networks has the same number of dependency relations. 


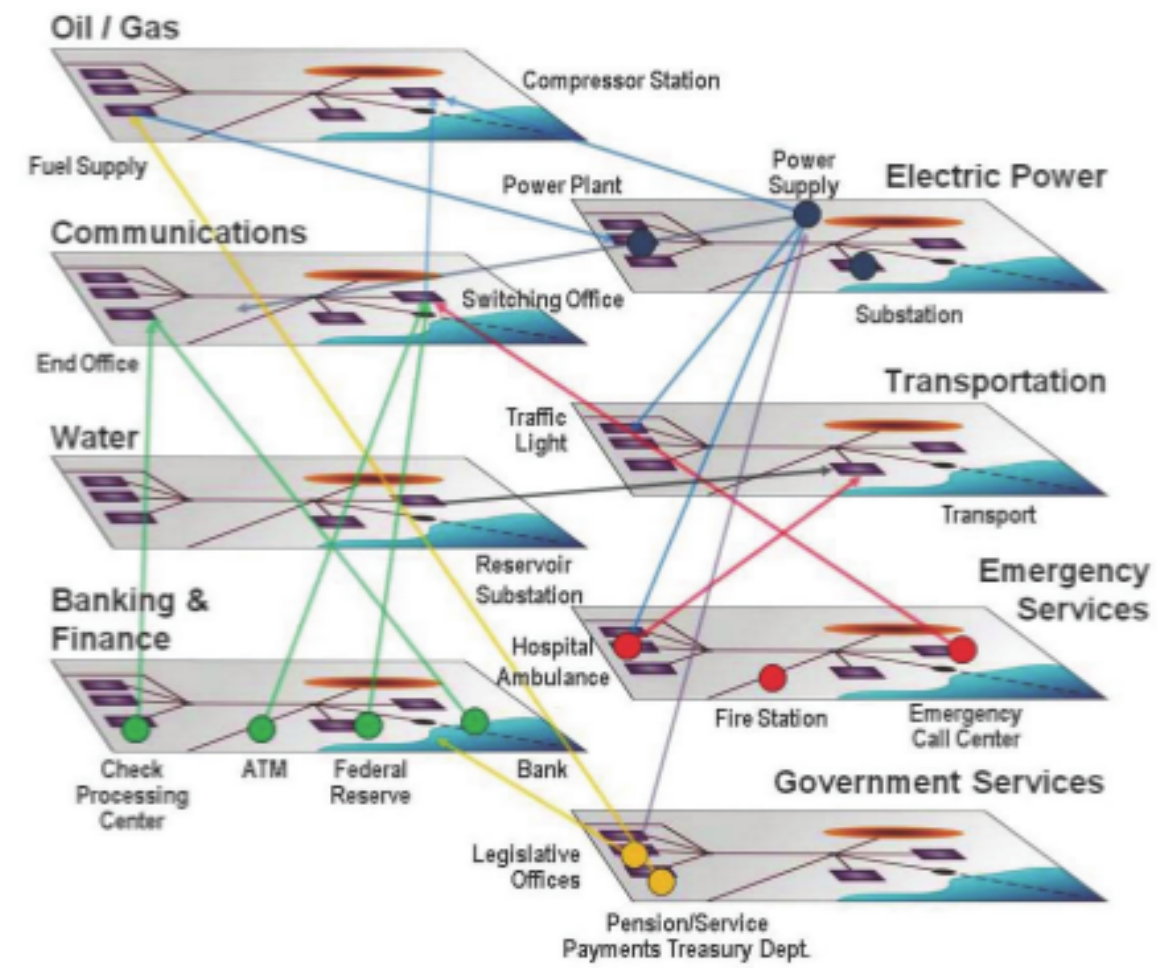

Figure 1: Modern infrastructure involves many dependency relations as shown in the Fig. from [44].

\section{Robustness of interdependent networks}

Just as earlier studies addressed the robustness of single networks using percolation methods [51, 52, 53, 54], similar methods, as well as other different methods, have been developed for interdependent networks $[39,40,55]$. When a fraction of nodes are removed from a network of interdependent networks, they cause dependent nodes in the other networks to fail [39]. The failure of these nodes will cause more nodes to become disconnected from the giant component, which will then cause other dependent nodes to fail. This process continues until a steady state is reached. Recent work examined the nature of this cascade and found that at criticality the length of the cascade has unique scaling properties [56]. Further, this cascade can be linked to other cascades which cause blackouts in power grids [20,57]. In real space, the cascade has characteristic spatial-temporal scales which can be modeled by a characteristic dependency length [58]. Due to the cascade, the removal of a single node can cause the system to collapse entirely, 


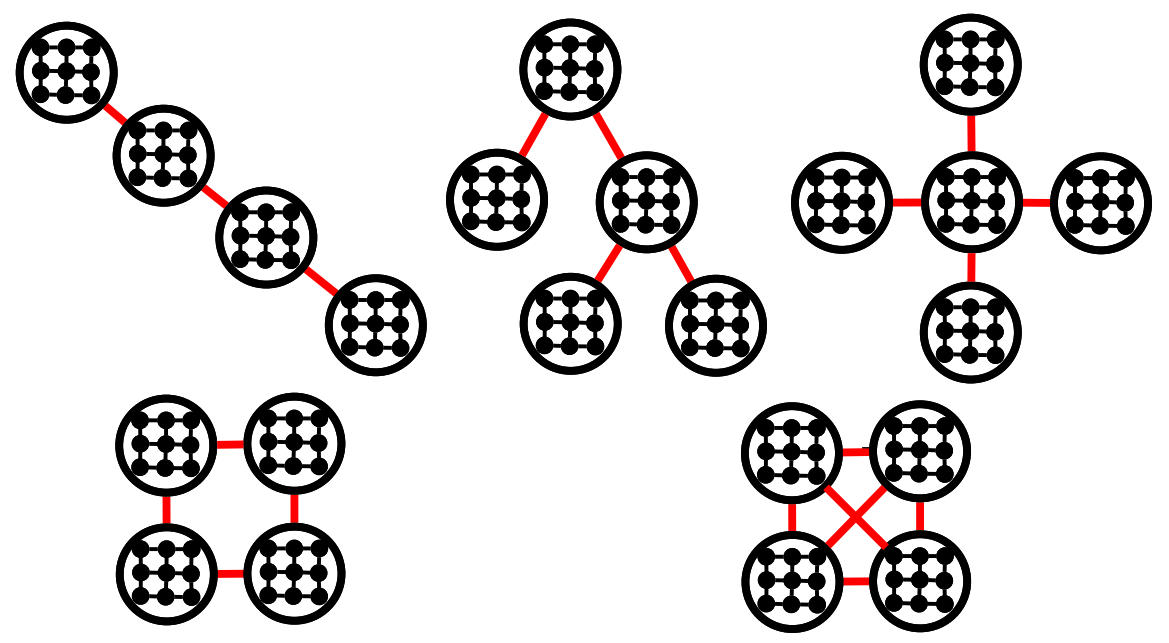

Figure 2: Networks of networks can assume many different structures defined based on which networks have dependency links between them. The top structures are all treelike structures whereas the bottom structures involve loops. Fig. after [50].

i.e. the transition is abrupt and first-order [40,59]. This discovery has significant implications to real world infrastructure as the discontinuity implies that a single additional failure can cause the total collapse of entire systems as was the case in the Northeastern United States Blackout in 2003 [60].

The initial work on the robustness of interdependent networks studied a pair of interdependent Erdôs-Rényi networks [39]. In that work, Buldyrev et al. [39] developed a theory based on percolation where the giant component was iteratively reduced due to the cascades. They then carried out numerical simulations to determine the size of the giant component at each step of the cascade, as well as the the giant component at the steady state, as a function of the fraction of initially removed nodes. We recall that for single Erdôs-Rényi networks the equation governing the size of the giant component is $P_{\infty}=p\left(1-e^{-k P_{\infty}}\right)$, where $k$ is the average degree of the network. In the case where $1-p$ nodes are removed from one network in a pair of interdependent Erdős-Rényi networks it was found $[39,61]$

$$
P_{\infty}=p\left(1-e^{-k P_{\infty}}\right)^{2} .
$$

In a series of papers by Gao et al. $[62,63,61,64]$ this framework was extended for various types of 
networks of networks that involve more than two networks. Simple analytic formulas were found for several cases. First for the case of $n$ interdependent Erdôs-Rényi networks whose dependencies (fully) form a tree (top structures in Fig. 2), Gao et al. [61] found that the size of the giant component is given by

$$
P_{\infty}=p\left(1-e^{-k P_{\infty}}\right)^{n}
$$

Even though networks are interdependent, it need not be the case that every node in the network has a dependent node in the other network. For example, a communications system could have its own autonomous power supply and other systems could also feature autonomous nodes. To account for this, researchers defined $q$ as the fraction of nodes in one network that have a dependent node in the second network [40]. The idea of partial dependency also leads to more structures that can be solved analytically, such as a loop of interdependent networks where each pair of networks has a fraction $q$ nodes interdependent. Gao et al. [61] found that for a loop of interdependent Erdős-Rényi networks,

$$
P_{\infty}=p\left(1-e^{-k P_{\infty}}\right)\left(1-q+q P_{\infty}\right)
$$

Note that when $q=0$ (no interdependence) this result reduces to the classical result for a single Erdős-Rényi network.

Another case that can be expressed analytically is the case where each network depends on exactly $m$ other networks, i.e. a random-regular network of networks (see the bottom structures in Fig. 2). Here too Gao et al. [62, 64] was able to solve this case for Erdôs-Rényi networks and obtained

$$
P_{\infty}=\frac{p}{2^{m}}\left(1-e^{-k P_{\infty}}\right)\left[1-q+\sqrt{(1-q)^{2}+4 q P_{\infty}}\right]^{m}
$$

Note here that when $m=0$ or $q=0$, Eq. (4) reduces to the result for single networks.

Other analytic results have been obtained by Bianconi et al. [65, 66], Baxter et al. [59, 67], and Cellai et al. [68]. 


\section{Interdependent networks with realistic features}

Other recent works, which are concisely reviewed below, have considered interdependent networks that have more realistic features.

\subsection{Realistic network structures}

First, several papers have examined interdependent networks that have more realistic degree distributions. For the case of interdependent scale-free networks, it was found that a broader degree distribution actually makes the networks less robust, in contradiction of the case for single networks $[39,69]$. Other studies have examined the influence of clustering $[70,71]$ and assortativity $[72,73]$ on interdependent networks.

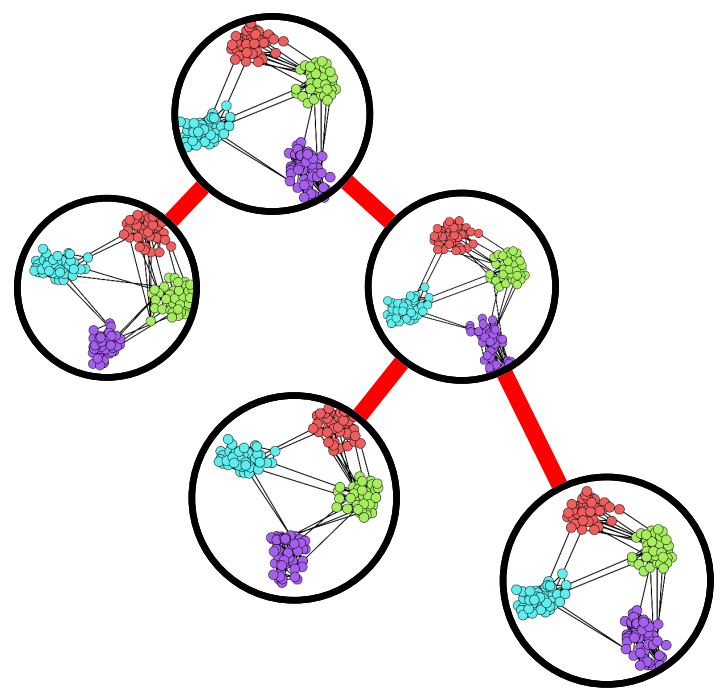

(a)

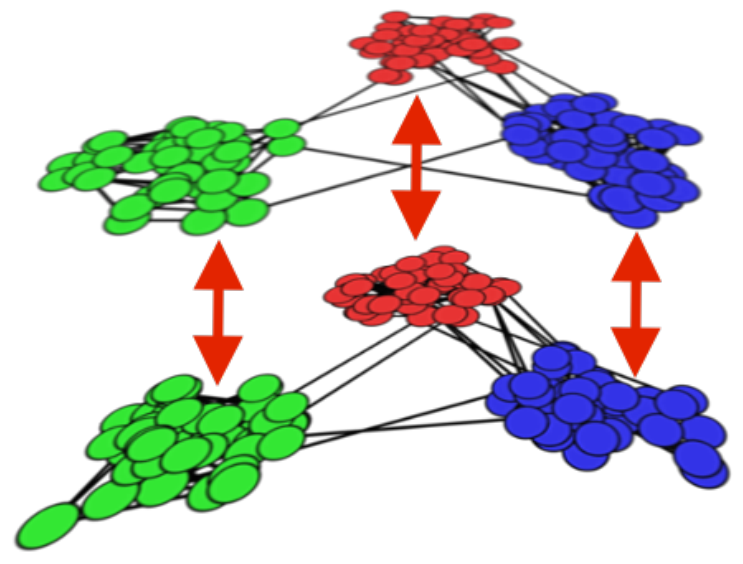

(b)

Figure 3: The model studied in [74]. (a) A treelike network of networks where each network has a modular structure. Dependency links are restricted such that they only connect nodes within the same communities, i.e a node in module $m_{a}$ in network $i$ will depend on a node also in module $m_{a}$ in network $j$. (b) Demonstration of the dependency relations between a pair of interdependent networks. Dependency links exist between nodes of the same color in different layers. Figure after [74].

Very recent work has also considered the effects of modularity or community structure on the robustness of single and interdependent networks [75, 74]. In those two works [75, 74], the researchers considered the case of attacks on the nodes that are interconnected, i.e. have at least one 
link to another community. It has been shown that interconnected nodes serve as efficient attack targets in many individual infrastructure networks, but especially in the Western U.S. power grid [76]. In [74] the authors considered the case of several interdependent networks where each network has the same number of modules of equal size. The dependency links were then restricted to be between nodes in the same community, but in different networks. It was noted that this model is realistic for infrastructure across cities, since each city has its own infrastructure and most of the interdependence is within a single city. Nonetheless, infrastructure networks are likely to be connected both within and across many cities. For example, a power grid and a communication network will likely encompass many cities, however it is likely that a power station and communication tower that are interdependent will be in the same city. A diagram of the model is shown in Fig. 3. It was found that for this model, as nodes are removed the system may undergo either one or two percolation transitions depending on the number of modules. If the system undergoes two transitions, the first transition occurs when the modules separate but are able to continue functioning individually. Only after further damage do the modules themselves also fail.

Another realistic structure for which particularly interesting effects were found is interdependent spatially embedded networks. Most critical infrastructures are embedded in space $[9,77,78]$ and thus understanding how spatial embedding affects the cascade of failures is very important. Spatially embedded networks have unique properties since most links will be of short length. For example, it is unlikely for two power stations on opposite sides of the United States will be connected, rather stations are usually connected to other nearby stations. This property, where nodes are connected to other nodes that are spatially nearby, is not present in random networks and leads to significantly different results. To model spatial networks, many researchers used 2D lattices and noted that any other embedded network will be in the same universality class $[79,26]$. One early work on interdependent spatially embedded networks considered varying $q$, the fraction of nodes which are interdependent. It was found analytically, that unlike random networks where a finite fraction of nodes must be interdependent in order for the transition to be first order, spatially 
embedded networks undergo a first order transition for any value of $q>0$ [80].

Another study considered the case of $q=1$, but where dependency links were restricted to be between nodes within some geographical distance, $r$. It was found that for low $r$ the percolation transition was continuous, but as $r$ increased, the transition became first order. The critical dependency length, where the transition becomes first-order occurs at $r_{c} \approx 8$ [81].

Later work considered combining $q$ and $r$, and found that as $q$ decreases, $r_{c}$ increases [82, 83]. Further work showed that when there are more than 2 interdependent networks, $r_{c}$ decreases [50]. Very recent work examined a more realistic spatial multiplex structure where interdependent nodes overlap $(r=0)$, but connectivity lengths are drawn from an exponential distribution [84].

\subsection{Realistic dependency relations}

Besides varying the structure of the networks themselves, it is also possible to place restrictions on the nature of the dependency links. For example, in the previous section we saw that for spatially embedded networks dependency links may be restricted to a finite length. Another realistic configuration of dependency links involves assigning high degree nodes in one network to be dependent on high degree nodes in the other network [85]. This makes sense since if a certain location is central then it is likely that both a power station and a communication tower located there will also be central. When dependency links are applied in such a way, interdependent scale-free networks become robust $[86,87,88]$. Many other studies have explored different variations of this idea $[73,89,87,90,91,92,93,68,94,95]$.

A recent development involved the recognition that it is often not sufficient to say whether a node is in the giant component or not, rather it also matters if the node participates in a process occurring on the network. A classic example is resistor networks. In these systems, nodes must not only be in the giant component, but also carry current in order for them to be considered functional (see Fig. 4) [96, 97, 98]. In the case of interdependent resistor networks, the nodes must both themselves carry current and have their dependent nodes carry current in order to be functional 


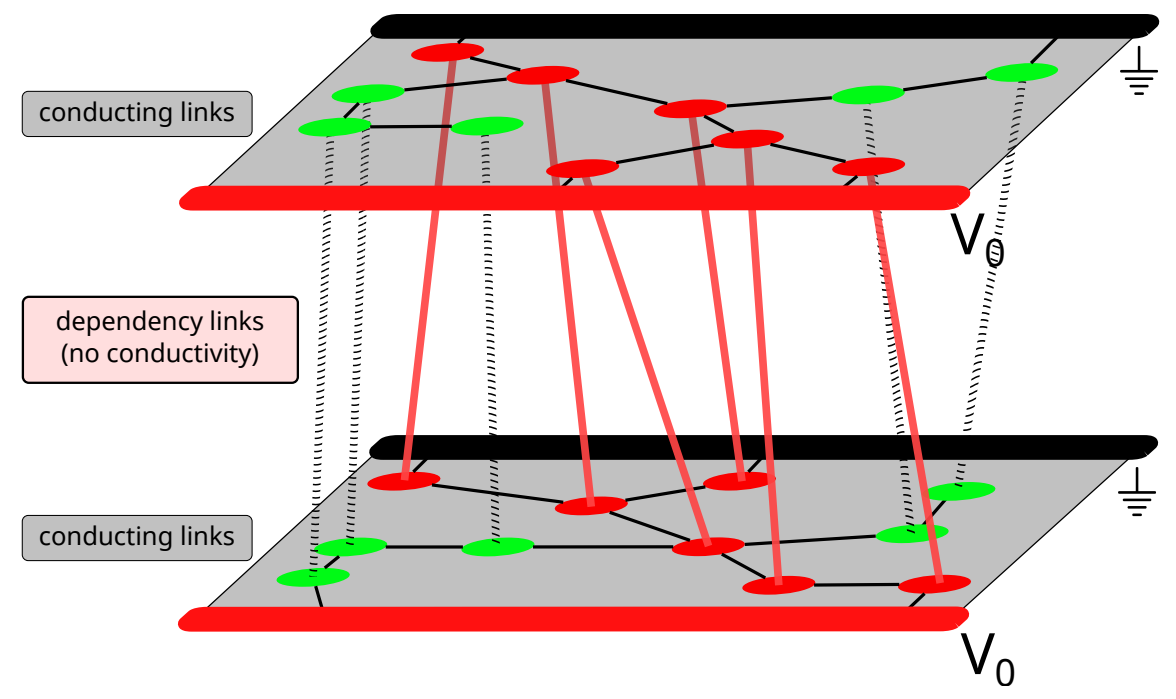

Figure 4: Two networks with process-based dependency are shown. Each network is connected to a source and a ground. In the figure, the red nodes are current-carrying nodes whereas the green nodes are dead ends. Nodes in one network are only considered functioning only if they carry current and the node they depend on carries current. Fig. from [99].

[99].

A study on interdependent resistor networks examined the fraction of nodes that carry current, as well as the overall conductivity of the system in the context of process based dependency [99]. Because functionality in this case relates to a microscopic physical process which can be studied in the laboratory, these results can be used to create new technologies such as a sensitive thermal or gas sensor.

\subsection{Localized attacks on interdependent networks}

A further aspect of interdependent networks where realistic features have been incorporated involves the nodes chosen to be removed. In other words, what happens if certain nodes are preferentially attacked? Initial studies examined targeted attacks on high-degree nodes in a pair of scale-free networks [100] and further work quickly expanded this to attacks on a more general network of networks [101].

More recent works have examined new types of attacks called localized attacks [102, 103]. In a study on a pair of interdependent spatially embedded networks, researchers examined what size 
of localized attack, i.e. 'hole,' must be made in a network in order for the cascade to lead to the collapse of the entire system. It was found that there is a metastable regime where even though the system is robust against random failures, it is vulnerable to geographically localized attacks. Further, regardless of the actual size of the system, the size of the 'hole,' denoted $r_{h}^{c}$, that must be made to collapse the system, remains fixed for a given degree and dependency length. This is in marked contrast to the case of a single lattice where the size of a hole must grow with the size of the system in order to lead to collapse. This sort of attack is very realistic in the context of an Electromagnetic Pulse (EMP) detonation. In Fig. 5 we determine the state of the system, i.e. stable, unstable, or metastable, according to system properties such as the degree of the system and the typical length of the dependency lengths. Given these two parameters for any real system, one can evaluate if the system is stable or vulnerable to either random or local failure. This information can help regulators allocate resources to make the system more resilient.

A further question related to attacks on interdependent networks involves determining the optimal way to prevent cascades of failures and avoid abrupt collapse. Aside from several suggestions mentioned earlier such as having high degree nodes depend on other high degree nodes, having short range dependency links, and increasing the number of autonomous nodes, there have been several more explicit studies on this question. These studies have examined which nodes should be made autonomous and other methods in order to increase the robustness of interdependent networks $[86,105]$

\section{Recovery in single networks and interdependent networks}

Despite efforts to build resilient infrastructure, there will always be situations where failures occur. In order to deal with this problem researchers have begun studying efficient methods to restore and recover interdependent networks. It was recently found [106] that spontaneous recovery can occur in single networks. This occurs when node recoveries are introduced in a system based on the Watts opinion model [107] and causes the network to fluctuate near its critical point between 


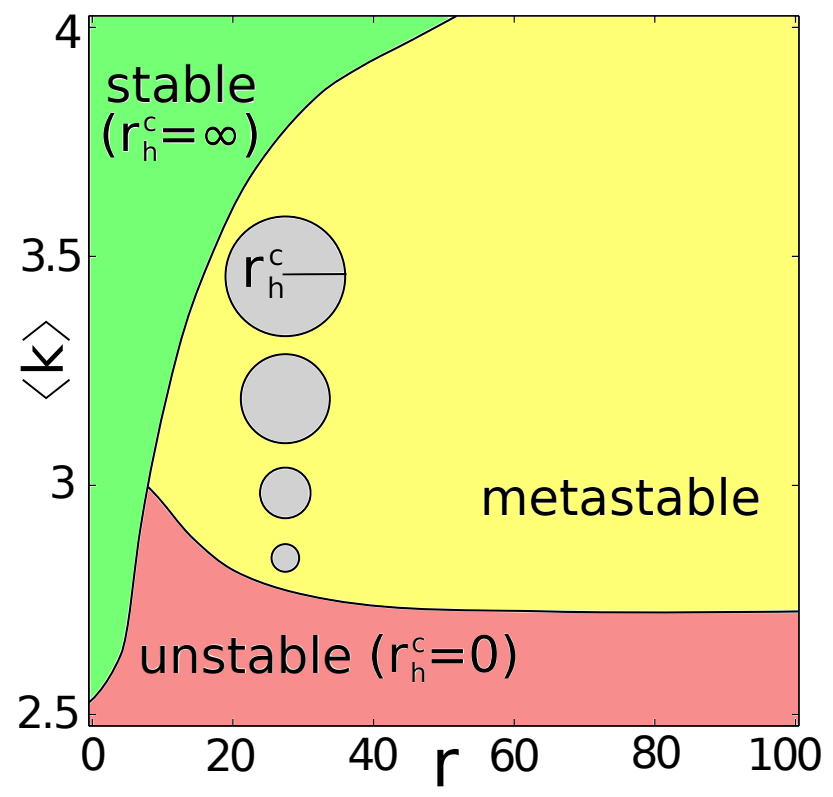

Figure 5: Here we show the phase diagram for localized attacks on interdependent spatial networks with an average degree $\langle k\rangle$ and dependency links of length $r$. Depending on these two parameters, the size needed for a hole to lead to the collapse of the entire system may be either $r_{h}^{c}=0$ (unstable case), $r_{h}^{c}=\infty$ (stable case) or $r_{h}^{c}$ finite (metastable case). In the metastable case a random attack on the network will not lead to its collapse, but a localized attack, even if it's a zero fraction of the system size, will cause the entire system to fail. The sizes of the circles represent the increase of the critical attack size, $r_{h}^{c}$, as $<k>$ increases (for fixed $r$ ). After [104].

active and inactive states.

A system of two interdependent networks has a much more complex phase diagram, characterized by two critical points and several spinodals (Fig. 6). These spinodals indicate the lines in parameter space, which if crossed lead the system to either fail to leave a collapsed state or recover to a functioning state, depending on the direction of the crossing. Given some level of failure in both networks, the optimal recovery strategy is to repair the fraction of nodes in each network that takes the shortest path in phase space towards the restoration spinodal [108]. Essentially this reveals how much effort to put into restoring each network in order to restore the overall functioning of the system of systems.

Several other approaches for restoration of interdependent network have also recently been proposed [109, 110]. 


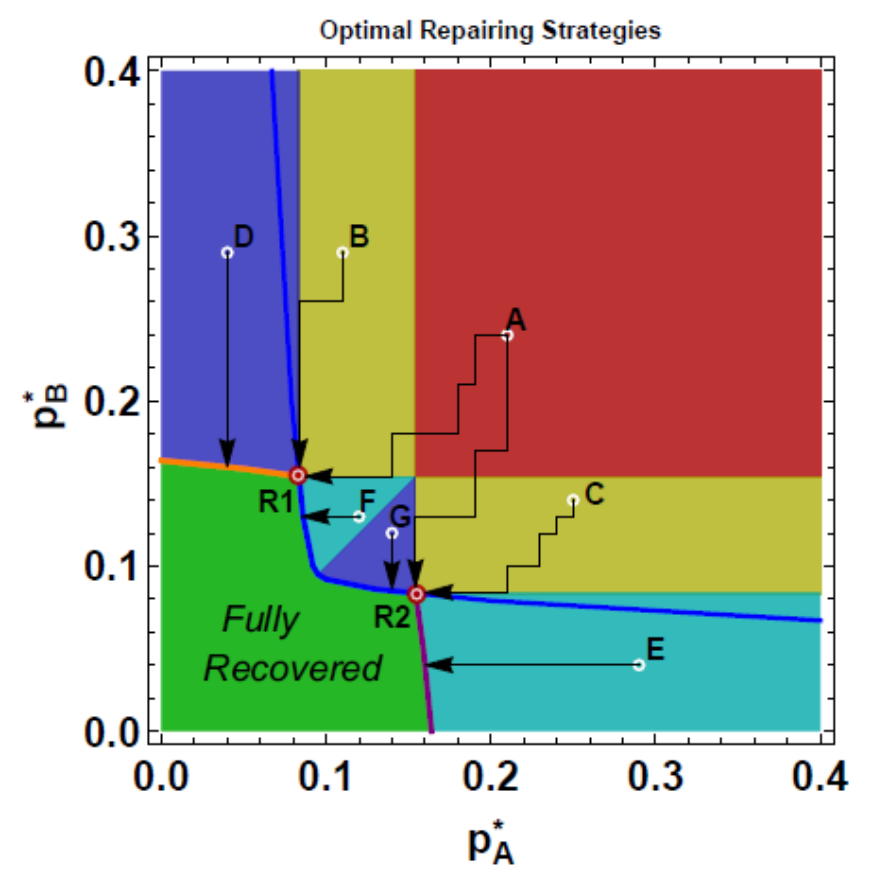

Figure 6: Optimal repairing strategies. The solution to the problem of optimal repairing corresponds to finding the minimal distance from the point where the collapsed system is situated to the border of the green region. In the red square region (point $\mathrm{A}$ for example) there are two solutions and it is equally optimal to reach any of the two spinodal crossing points R1 and R2 by decreasing $p_{A}$ and $p_{B}$, the probabilities of failure in networks $A$ and $B$ respectively. In the yellow regions, it is optimal to reach only one triple point, namely $\mathrm{R} 1$ for the sector containing point $\mathrm{B}$ and $\mathrm{R} 2$ for the sector containing point $\mathrm{C}$. In the dark blue regions such as the regions containing points $\mathrm{D}$ and $\mathrm{G}$, it is optimal to make repairs only in network $B$ in order to cross the orange line (for point $\mathrm{D}$ ) or the blue line (for point $\mathrm{G}$ ). In the light blue regions such as the regions containing points $\mathrm{E}$ and $\mathrm{F}$, it is optimal to make repairs only in network $A$ in order to cross the maroon line (for point $\mathrm{E}$ ) or the blue line (for point F). We extend the blue line which represents one of the crossing regions in order to highlight that spinodal crossing points represent the solution of the optimal repairing for the warm color regions (red and yellow). After [108]. 


\section{Conclusion}

There continue to be new developments in the understanding of interdependent networks. The recognition of the importance of analyzing the relations between systems is becoming more and more crucial as we move towards all sorts of smart technologies, like SMART grids, SMART cities, and the internet of things (IOT). Furthermore, interdependent networks have found significant applications outside of infrastructure in areas like finance [111, 112] and climate studies [113]. Further studies on the robustness of interdependent networks, recovery in interdependent networks, and other new aspects of interdependent networks will likely lead to more new discoveries of other rich phenomenon.

\section{Acknowledgments}

We acknowledge the MULTIPLEX (No. 317532) EU project, the Deutsche Forschungsgemeinschaft (DFG), the Israel Science Foundation, ONR and DTRA for financial support. M.D. thanks the Azrieli Foundation for the award of an Azrieli Fellowship grant.

[1] A.-L. Barabási, Scale-free networks: a decade and beyond, Science 325 (5939) (2009) 412413.

[2] A.-L. Barabási, R. Albert, Emergence of Scaling in Random Networks, Science 286 (5439) (1999) 509-512. doi : 10.1126/science.286.5439.509.

URL http: //wwW . sciencemag . org/content/286/5439/509 . abstract

[3] G. Caldarelli, Scale-free networks: complex webs in nature and technology, Oxford University Press, 2007.

[4] D. J. Watts, S. H. Strogatz, Collective dynamics of 'small-world' networks, Nature 393 (6684) (1998) 440-442. doi : 10 . 1038/30918.

URL http: //dx . doi .org/10 . 1038/30918 
[5] M. Girvan, M. E. Newman, Community structure in social and biological networks, Proceedings of the National Academy of Sciences 99 (12) (2002) 7821-7826.

[6] P. J. Mucha, T. Richardson, K. Macon, M. A. Porter, J.-P. Onnela, Community structure in time-dependent, multiscale, and multiplex networks, science 328 (5980) (2010) 876-878.

[7] R. Pastor-Satorras, A. Vázquez, A. Vespignani, Dynamical and correlation properties of the internet, Physical Review Letters 87 (25) (2001) 258701.

[8] M. E. Newman, Assortative mixing in networks, Physical Review Letters 89 (20) (2002) 208701.

[9] M. Barthélemy, Spatial networks, Physics Reports 499 (1) (2011) 1-101.

[10] D. Brockmann, D. Helbing, The hidden geometry of complex, network-driven contagion phenomena, Science 342 (6164) (2013) 1337-1342.

[11] L. Hufnagel, D. Brockmann, T. Geisel, Forecast and control of epidemics in a globalized world, Proceedings of the National Academy of Sciences of the United States of America 101 (42) (2004) 15124-15129.

[12] R. Pastor-Satorras, A. Vespignani, Epidemic spreading in scale-free networks, Physical Review Letters 86 (14) (2001) 3200.

[13] M. E. Newman, Spread of epidemic disease on networks, Physical Review E 66 (1) (2002) 016128.

[14] R. Cohen, S. Havlin, D. Ben-Avraham, Efficient immunization strategies for computer networks and populations, Physical Review Letters 91 (24) (2003) 247901.

[15] J. F. Donges, Y. Zou, N. Marwan, J. Kurths, The backbone of the climate network, EPL (Europhysics Letters) 87 (4) (2009) 48007.

URL http: //stacks . iop.org/0295-5075/87/i=4/a=48007 
[16] K. Yamasaki, A. Gozolchiani, S. Havlin, Climate networks around the globe are significantly affected by el nino, Physical Review Letters 100 (22) (2008) 228501.

[17] G. Bonanno, G. Caldarelli, F. Lillo, S. Miccichè, N. Vandewalle, R. N. Mantegna, Networks of equities in financial markets, The European Physical Journal B-Condensed Matter and Complex Systems 38 (2) (2004) 363-371.

[18] X. Zhang, S. Shao, H. E. Stanley, S. Havlin, Dynamic motifs in socio-economic networks, EPL (Europhysics Letters) 108 (5) (2014) 58001.

[19] G. Kossinets, D. J. Watts, Empirical analysis of an evolving social network, Science 311 (5757) (2006) 88-90.

[20] A. E. Motter, Y.-C. Lai, Cascade-based attacks on complex networks, Physical Review E $66(6)(2002) 065102$.

[21] D. Li, B. Fu, Y. Wang, G. Lu, Y. Berezin, H. E. Stanley, S. Havlin, Percolation transition in dynamical traffic network with evolving critical bottlenecks, Proceedings of the National Academy of Sciences 112 (3) (2015) 669-672. doi : 10.1073/pnas . 1419185112.

URL http: //wwW . pnas . org/content/112/3/669. abstract

[22] A. Bashan, R. P. Bartsch, J. W. Kantelhardt, S. Havlin, P. C. Ivanov, Network physiology reveals relations between network topology and physiological function, Nature communications 3 (2012) 702.

[23] D. S. Bassett, E. Bullmore, Small-world brain networks, The neuroscientist 12 (6) (2006) 512-523.

[24] L. K. Gallos, H. A. Makse, M. Sigman, A small world of weak ties provides optimal global integration of self-similar modules in functional brain networks, Proceedings of the National Academy of Sciences 109 (8) (2012) 2825-2830. 
[25] D. Stauffer, A. Aharony, Introduction to Percolation Theory, Taylor \& Francis, 1994. URL http://books.google.co.il/books?id=v66plleij5QC

[26] A. Bunde, S. Havlin, Fractals and disordered systems, Springer-Verlag New York, Inc., 1991.

[27] H. Stanley, Introduction to Phase Transitions and Critical Phenomena, International series of monographs on physics, Oxford University Press, 1971.

[28] B. Bollobás, Modern graph theory, Vol. 184, Springer Science \& Business Media, 1998.

[29] P. Erdős, A. Rényi, On random graphs i, Publ. Math. Debrecen 6 (1959) 290-297.

[30] P. Erdős, A. Rényi, On the strength of connectedness of a random graph, Acta Mathematica Hungarica 12 (1-2) (1961) 261-267.

[31] R. Cohen, K. Erez, D. ben Avraham, S. Havlin, Resilience of the Internet to Random Breakdowns, Phys. Rev. Lett. 85 (2000) 4626-4628. doi : 10. 1103/PhysRevLett . 85 . 4626. URL http://link.aps .org/doi/10.1103/PhysRevLett . 85.4626

[32] M. Kivelä, A. Arenas, M. Barthélémy, J. P. Gleeson, Y. Moreno, M. A. Porter, Multilayer networks, Journal of Complex Networks 2 (3) (2014) 203-271. doi : 10. 1093/comnet/ cnuQ16.

URL http: //comnet . oxfordjournals . org/content/2/3/203 . abstract

[33] G. Bianconi, Statistical mechanics of multiplex networks: Entropy and overlap, Phys. Rev. E 87 (2013) 062806. doi : 10.1103/PhysRevE. 87.062806.

URL http://link .aps .org/doi/10.1103/PhysRevE.87.062806

[34] S. Boccaletti, G. Bianconi, R. Criado, C. Del Genio, J. Gómez-Gardeñes, M. Romance, I. Sendina-Nadal, Z. Wang, M. Zanin, The structure and dynamics of multilayer networks, 
Physics Reportsdoi : 10.1016/j . physrep.2014.07.001.

URL http://dx.doi .org/10.1016/j .physrep.2014.07.001

[35] A. Vespignani, Complex networks: The fragility of interdependency, Nature 464 (7291) (2010) 984-985. doi : 10.1038/464984a.

URL http://dx.doi.org/10.1038/464984a

[36] S. Rinaldi, J. Peerenboom, T. Kelly, Identifying, understanding, and analyzing critical infrastructure interdependencies, Control Systems, IEEE 21 (6) (2001) 11-25. doi : $10.1109 / 37.969131$.

[37] E. A. Leicht, R. M. D'Souza, Percolation on interacting networks, ArXiv e-printsarXiv: 0907.0894.

[38] K.-M. Lee, B. Min, K.-I. Goh, Towards real-world complexity: an introduction to multiplex networks, The European Physical Journal B 88 (2) (2015) 1-20.

[39] S. V. Buldyrev, R. Parshani, G. Paul, H. E. Stanley, S. Havlin, Catastrophic cascade of failures in interdependent networks, Nature 464 (7291) (2010) 1025-1028. doi : 10.1038/ nature08932.

URL http://dx.doi.org/10.1038/nature08932

[40] R. Parshani, S. V. Buldyrev, S. Havlin, Interdependent Networks: Reducing the Coupling Strength Leads to a Change from a First to Second Order Percolation Transition, Phys. Rev. Lett. 105 (2010) 048701. doi : 10.1103/PhysRevLett. 105 . 048701.

URL http://link. aps.org/doi/10.1103/PhysRevLett. 105.048701

[41] Y. Hu, S. Havlin, H. A. Makse, Conditions for viral influence spreading through multiplex correlated social networks, Physical Review X 4 (2) (2014) 021031. 
[42] S. Gómez, A. Díaz-Guilera, J. Gómez-Gardeñes, C. J. Pérez-Vicente, Y. Moreno, A. Arenas, Diffusion Dynamics on Multiplex Networks, Phys. Rev. Lett. 110 (2013) 028701. doi : 10.1103/PhysRevLett. 110.028701 .

URL http://link.aps.org/doi/10.1103/PhysRevLett. 110.028701

[43] M. De Domenico, A. Solé-Ribalta, E. Cozzo, M. Kivelä, Y. Moreno, M. A. Porter, S. Gómez, A. Arenas, Mathematical formulation of multilayer networks, Physical Review X 3 (4) (2013) 041022.

[44] J. S. Foster Jr, E. Gjelde, W. R. Graham, R. J. Hermann, H. M. Kluepfel, R. L. Lawson, G. K. Soper, L. L. Wood, J. B. Woodard, Report of the commission to assess the threat to the united states from electromagnetic pulse (emp) attack: Critical national infrastructures, Tech. rep., DTIC Document (2008).

[45] R. Parshani, S. V. Buldyrev, S. Havlin, Critical effect of dependency groups on the function of networks, Proceedings of the National Academy of Sciences 108 (3) (2011) 1007-1010. doi : 10.1073/pnas. 1008404108 .

URL http: //wwW . pnas . org/content/108/3/1007 . abstract

[46] A. Bashan, R. Parshani, S. Havlin, Percolation in networks composed of connectivity and dependency links, Phys. Rev. E 83 (2011) 051127. doi : 10.1103/PhysRevE . 83.051127. URL http://link.aps.org/doi/10.1103/PhysRevE.83.051127

[47] S.-W. Son, G. Bizhani, C. Christensen, P. Grassberger, M. Paczuski, Percolation theory on interdependent networks based on epidemic spreading, EPL (Europhysics Letters) 97 (1) (2012) 16006.

URL http: //stacks . iop.org/0295-5075/97/i=1/a=16006

[48] J. Shao, S. V. Buldyrev, S. Havlin, H. E. Stanley, Cascade of failures in coupled network systems with multiple support-dependence relations, Phys. Rev. E 83 (2011) 036116. doi : 
10.1103/PhysRevE.83.036116.

URL http: //link .aps .org/doi/10.1103/PhysRevE. 83.036116

[49] M. M. Danziger, A. Bashan, Y. Berezin, L. M. Shekhtman, S. Havlin, An introduction to interdependent networks, in: Nonlinear Dynamics of Electronic Systems, Springer, 2014, pp. 189-202.

[50] L. M. Shekhtman, Y. Berezin, M. M. Danziger, S. Havlin, Robustness of a network formed of spatially embedded networks, Phys. Rev. E 90 (2014) 012809. doi : 10.1103/ PhysRevE.90.012809.

URL http: //link.aps.org/doi/10.1103/PhysRevE.90.012809

[51] R. Cohen, S. Havlin, Complex Networks: Structure, Robustness and Function, Cambridge University Press, 2010.

[52] D. S. Callaway, M. E. J. Newman, S. H. Strogatz, D. J. Watts, Network Robustness and Fragility: Percolation on Random Graphs, Phys. Rev. Lett. 85 (2000) 5468-5471. doi : 10.1103/PhysRevLett. 85.5468 .

URL http://link.aps.org/doi/10.1103/PhysRevLett.85.5468

[53] M. E. J. Newman, I. Jensen, R. M. Ziff, Percolation and epidemics in a two-dimensional small world, Phys. Rev. E 65 (2002) 021904. doi : 10.1103/PhysRevE. 65 . 021904. URL http: //link.aps .org/doi/10.1103/PhysRevE.65.021904

[54] M. Newman, Networks: an introduction, OUP Oxford, 2010.

[55] F. Radicchi, Percolation in real interdependent networks, Nature Physics 11 (7) (2015) 597602. doi : 10.1038/nphys3374.

URL http: //dx . doi .org/10.1038/nphys3374 
[56] D. Zhou, A. Bashan, R. Cohen, Y. Berezin, N. Shnerb, S. Havlin, Simultaneous first- and second-order percolation transitions in interdependent networks, Phys. Rev. E 90 (2014) 012803. doi : 10.1103/PhysRevE. 90.012803.

URL http: //link.aps .org/doi/10.1103/PhysRevE.90.012803

[57] I. Dobson, B. A. Carreras, V. E. Lynch, D. E. Newman, Complex systems analysis of series of blackouts: Cascading failure, critical points, and self-organization, Chaos: An Interdisciplinary Journal of Nonlinear Science 17 (2) (2007) 026103. doi : 10 . 1063/1 . 2737822. URL http: //link . aip .org/link/?CHA/17/026103/1

[58] J. Zhao, D. Li, H. Sanhedrai, R. Cohen, S. Havlin, Spatio-temporal propagation of cascading overload failures, Nature Communications. In Press.

[59] G. J. Baxter, S. N. Dorogovtsev, A. V. Goltsev, J. F. F. Mendes, Avalanche Collapse of Interdependent Networks, Phys. Rev. Lett. 109 (2012) 248701. doi : 10.1103/PhysRevLett. 109.248701 .

URL http: //link.aps .org/doi/10.1103/PhysRevLett. 109.248701

[60] New York Independent System Operator (NYISO) Interim Report on the August 14, 2003 Blackout.

[61] J. Gao, S. V. Buldyrev, S. Havlin, H. E. Stanley, Robustness of a Network of Networks, Phys. Rev. Lett. 107 (2011) 195701. doi : 10.1103/PhysRevLett. 107.195701.

URL http://link.aps .org/doi/10.1103/PhysRevLett. 107.195701

[62] J. Gao, S. V. Buldyrev, H. E. Stanley, X. Xu, S. Havlin, Percolation of a general network of networks, Phys. Rev. E 88 (2013) 062816. doi : 10.1103/PhysRevE. 88.062816. URL http://link.aps.org/doi/10.1103/PhysRevE.88.062816

[63] J. Gao, S. V. Buldyrev, S. Havlin, H. E. Stanley, Robustness of a network formed by $n$ interdependent networks with a one-to-one correspondence of dependent nodes, Phys. Rev. 
E 85 (2012) 066134. doi : 10.1103/PhysRevE. 85.066134.

URL http://link. aps.org/doi/10.1103/PhysRevE. 85.066134

[64] J. Gao, S. V. Buldyrev, H. E. Stanley, S. Havlin, Networks formed from interdependent networks, Nature Physics 8 (1) (2012) 40-48. doi : 10. 1038/nphys2180.

URL http://dx.doi .org/10.1038/nphys2180

[65] G. Bianconi, S. N. Dorogovtsev, J. F. Mendes, Mutually connected component of networks of networks with replica nodes, Physical Review E 91 (1) (2015) 012804.

[66] G. Bianconi, S. N. Dorogovtsev, Multiple percolation transitions in a configuration model of a network of networks, Physical Review E 89 (6) (2014) 062814.

[67] G. Baxter, S. Dorogovtsev, A. Goltsev, J. Mendes, Avalanches in multiplex and interdependent networks, in: G. D’Agostino, A. Scala (Eds.), Networks of Networks: The Last Frontier of Complexity, Understanding Complex Systems, Springer International Publishing, 2014, pp. 37-52. doi : 10.1007/978-3-319-03518-5_2.

URL http://dx.doi .org/10.1007/978-3-319-03518-5_2

[68] D. Cellai, E. López, J. Zhou, J. P. Gleeson, G. Bianconi, Percolation in multiplex networks with overlap, Phys. Rev. E 88 (2013) 052811. doi : 10.1103/PhysRevE. 88.052811.

URL http://link . aps .org/doi/10.1103/PhysRevE. 88.052811

[69] D. Zhou, J. Gao, H. E. Stanley, S. Havlin, Percolation of partially interdependent scale-free networks, Phys. Rev. E 87 (2013) 052812. doi : 10.1103/PhysRevE . 87.052812.

URL http://link.aps.org/doi/10.1103/PhysRevE. 87.052812

[70] S. Shao, X. Huang, H. E. Stanley, S. Havlin, Robustness of a partially interdependent network formed of clustered networks, Phys. Rev. E 89 (2014) 032812. doi:10.1103/ PhysRevE. 89.032812.

URL http://link.aps.org/doi/10.1103/PhysRevE.89.032812 
[71] Huang, Xuqing, Shao, Shuai, Wang, Huijuan, Buldyrev, Sergey V., Eugene Stanley, H., Havlin, Shlomo, The robustness of interdependent clustered networks, EPL 101 (1) (2013) 18002. doi : 10.1209/0295-5075/101/18002.

URL http://dx.doi.org/10.1209/0295-5075/101/18002

[72] D. Zhou, H. E. Stanley, G. D'Agostino, A. Scala, Assortativity decreases the robustness of interdependent networks, Phys. Rev. E 86 (2012) 066103. doi : 10.1103/PhysRevE. 86. 066103.

URL http://link.aps.org/doi/10.1103/PhysRevE. 86.066103

[73] S. Watanabe, Y. Kabashima, Cavity-based robustness analysis of interdependent networks: Influences of intranetwork and internetwork degree-degree correlations, Phys. Rev. E 89 (2014) 012808. doi : 10.1103/PhysRevE. 89.012808.

URL http://link.aps.org/doi/10.1103/PhysRevE. 89.012808

[74] L. M. Shekhtman, S. Shai, S. Havlin, Resilience of networks formed of interdependent modular networks, New Journal of Physics 17 (12) (2015) 123007.

URL http://stacks . iop.org/1367-2630/17/i=12/a=123007

[75] S. Shai, D. Y. Kenett, Y. N. Kenett, M. Faust, S. Dobson, S. Havlin, Critical tipping point distinguishing two types of transitions in modular network structures, Phys. Rev. E 92 (2015) 062805. doi: 10.1103/PhysRevE.92.062805.

URL http://link.aps.org/doi/10.1103/PhysRevE. 92.062805

[76] B. R. da Cunha, J. C. González-Avella, S. Gonçalves, Fast fragmentation of networks using module-based attacks, PloS one 10 (11).

[77] P. Hines, S. Blumsack, E. Cotilla Sanchez, C. Barrows, The Topological and Electrical Structure of Power Grids, in: System Sciences (HICSS), 2010 43rd Hawaii International Conference on, 2010, pp. 1-10. doi: 10.1109/HICSS.2010.398. 
[78] P. Hines, S. Blumsack, E. Cotilla Sanchez, C. Barrows, The topological and electrical structure of power grids, in: System Sciences (HICSS), 2010 43rd Hawaii International Conference on, IEEE, 2010, pp. 1-10.

[79] L. Daqing, K. Kosmidis, A. Bunde, S. Havlin, Dimension of spatially embedded networks, Nature Physics 7 (6) (2011) 481-484. doi : 10. 1038/nphys 1932.

URL http://dx.doi .org/10.1038/nphys 1932

[80] A. Bashan, Y. Berezin, S. V. Buldyrev, S. Havlin, The extreme vulnerability of interdependent spatially embedded networks, Nature Physics 9 (2013) 667-672. doi : 10 . 1038/ nphys 2727.

URL http://dx.doi .org/10.1038/nphys2727

[81] W. Li, A. Bashan, S. V. Buldyrev, H. E. Stanley, S. Havlin, Cascading Failures in Interdependent Lattice Networks: The Critical Role of the Length of Dependency Links, Phys. Rev. Lett. 108 (2012) 228702. doi : 10.1103/PhysRevLett. 108. 228702.

URL http://link.aps .org/doi/10.1103/PhysRevLett.108.228702

[82] M. M. Danziger, A. Bashan, Y. Berezin, S. Havlin, Percolation and cascade dynamics of spatial networks with partial dependency, Journal of Complex Networks 2 (4) (2014) 460474. doi:10.1093/comnet/cnu020.

URL http://comnet . oxfordjournals . org/content/2/4/460. abstract

[83] M. M. Danziger, A. Bashan, Y. Berezin, S. Havlin, Interdependent spatially embedded networks: Dynamics at percolation threshold, in: Signal-Image Technology Internet-Based Systems (SITIS), 2013 International Conference on, 2013, pp. 619-625. doi:10.1109/ SITIS.2013.101.

[84] M. M. Danziger, L. M. Shekhtman, Y. Berezin, S. Havlin, Two distinct transitions in spatially embedded multiplex networks, arXiv preprint arXiv:1505.01688. 
[85] B. Min, S. Do Yi, K.-M. Lee, K.-I. Goh, Network robustness of multiplex networks with interlayer degree correlations, Physical Review E 89 (4) (2014) 042811.

[86] C. M. Schneider, N. Yazdani, N. A. Araújo, S. Havlin, H. J. Herrmann, Towards designing robust coupled networks, Scientific reports 3.

[87] R. Parshani, C. Rozenblat, D. Ietri, C. Ducruet, S. Havlin, Inter-similarity between coupled networks, EPL (Europhysics Letters) 92 (6) (2010) 68002.

URL http: //stacks . iop. org/0295-5075/92/i=6/a=68002

[88] C. Buono, L. G. Alvarez-Zuzek, P. A. Macri, L. A. Braunstein, Epidemics in partially overlapped multiplex networks, PloS one 9 (3) (2014) 5.

[89] Y. Hu, D. Zhou, R. Zhang, Z. Han, C. Rozenblat, S. Havlin, Percolation of interdependent networks with intersimilarity, Phys. Rev. E 88 (2013) 052805. doi : 10.1103/PhysRevE. 88.052805 .

URL http://link.aps .org/doi/10.1103/PhysRevE. 88.052805

[90] M. Li, R.-R. Liu, C.-X. Jia, B.-H. Wang, Critical effects of overlapping of connectivity and dependence links on percolation of networks, New Journal of Physics 15 (9) (2013) 093013. URL http: //stacks . iop.org/1367-2630/15/i=9/a=093013

[91] S. V. Buldyrev, N. W. Shere, G. A. Cwilich, Interdependent networks with identical degrees of mutually dependent nodes, Phys. Rev. E 83 (2011) 016112. doi : 10.1103/PhysRevE. 83. 016112 .

URL http://link.aps.org/doi/10.1103/PhysRevE . 83.016112

[92] Y. Kornbluth, S. Lowinger, G. Cwilich, S. V. Buldyrev, Cascading failures in networks with proximate dependent nodes, Physical Review E 89 (3) (2014) 032808. 
[93] L. D. Valdez, P. A. Macri, H. E. Stanley, L. A. Braunstein, Triple point in correlated interdependent networks, Phys. Rev. E 88 (2013) 050803. doi : 10.1103/PhysRevE . 88 . 050803. URL http: //link.aps .org/doi/10.1103/PhysRevE . 88.050803

[94] B. Min, S. Lee, K.-M. Lee, K.-I. Goh, Link overlap, viability, and mutual percolation in multiplex networks, Chaos, Solitons \& Fractals 72 (2015) 49-58.

[95] J. Y. Kim, K.-I. Goh, Coevolution and Correlated Multiplexity in Multiplex Networks, Phys. Rev. Lett. 111 (2013) 058702. doi : 10.1103/PhysRevLett. 111.058702.

URL http: //link.aps .org/doi/10.1103/PhysRevLett. 111.058702

[96] S. Kirkpatrick, Percolation and conduction, Rev. Mod. Phys. 45 (1973) 574-588. doi : 10.1103/RevModPhys . 45.574 .

URL http: //link . aps .org/doi/10.1103/RevModPhys . 45. 574

[97] L. d. Arcangelis, S. Redner, A. Coniglio, Anomalous voltage distribution of random resistor networks and a new model for the backbone at the percolation threshold, Phys. Rev. B 31 (1985) 4725-4727. doi : 10.1103/PhysRevB . 31 . 4725.

URL http: //link.aps .org/doi/10.1103/PhysRevB. 31.4725

[98] Y. Strelniker, R. Berkovits, A. Frydman, S. Havlin, Percolation transition in a twodimensional system of ni granular ferromagnets, Phys. Rev. E 69 (2004) 065105. doi : 10.1103/PhysRevE.69.065105.

URL http: //link.aps.org/doi/10.1103/PhysRevE.69.065105

[99] M. M. Danziger, A. Bashan, S. Havlin, Interdependent resistor networks with process-based dependency, New Journal of Physics 17 (4) (2015) 043046. doi : 10. 1088/1367-2630/ $17 / 4 / 043046$.

URL http : //stacks . iop . org/1367-2630/17/i=4/a=043046 
[100] X. Huang, J. Gao, S. V. Buldyrev, S. Havlin, H. E. Stanley, Robustness of interdependent networks under targeted attack, Phys. Rev. E 83 (2011) 065101. doi : 10 . 1103/PhysRevE. 83.065101.

URL http: //link.aps .org/doi/10.1103/PhysRevE. 83.065101

[101] G. Dong, J. Gao, R. Du, L. Tian, H. E. Stanley, S. Havlin, Robustness of network of networks under targeted attack, Phys. Rev. E 87 (2013) 052804. doi : 10.1103/PhysRevE. 87.052804

URL http://link.aps .org/doi/10.1103/PhysRevE.87.052804

[102] S. Shao, X. Huang, H. E. Stanley, S. Havlin, Percolation of localized attack on complex networks, New Journal of Physics 17 (2) (2015) 023049.

[103] X. Yuan, S. Shao, H. E. Stanley, S. Havlin, How breadth of degree distribution influences network robustness: Comparing localized and random attacks, Physical Review E 92 (3) (2015) 032122.

[104] Y. Berezin, A. Bashan, M. M. Danziger, D. Li, S. Havlin, Localized attacks on spatially embedded networks with dependencies, Scientific Reports 5. doi : 10. 1038/srep08934. URL http://dx .doi .org/10.1038/srep08934

[105] L. D. Valdez, P. A. Macri, L. A. Braunstein, A triple point induced by targeted autonomization on interdependent scale-free networks, Journal of Physics A: Mathematical and Theoretical 47 (5) (2014) 055002.

URL http: //stacks . iop.org/1751-8121/47/i=5/a=055002

[106] A. Majdandzic, B. Podobnik, S. V. Buldyrev, D. Y. Kenett, S. Havlin, H. Eugene Stanley, Spontaneous recovery in dynamical networks, Nature Physics 10 (1) (2013) 3438. doi : 10. $1038 /$ nphys 2819 .

URL http://dx . doi .org/10.1038/nphys2819 
[107] D. J. Watts, A simple model of global cascades on random networks, Proceedings of the National Academy of Sciences 99 (9) (2002) 5766-5771.

[108] A. Majdandzic, L. A. Braunstein, C. Curme, I. Vodenska, S. Levy-Carciente, H. E. Stanley, S. Havlin, Multiple tipping points and optimal repairing in interacting networks, Nature Comm. in press.

[109] M. Di Muro, C. La Rocca, H. Stanley, S. Havlin, L. Braunstein, Recovery of interdependent networks, arXiv preprint arXiv:1512.02555.

[110] M. Stippinger, J. Kertész, Enhancing resilience of interdependent networks by healing, Physica A: Statistical Mechanics and its Applications 416 (0) (2014) 481 - 487. doi : http://dx.doi .org/10.1016/j.physa.2014.08.069.

URL http://wWW.sciencedirect.com/science/article/pii/ SQ378437114007547

[111] D. Y. Kenett, S. Havlin, Network science: a useful tool in economics and finance, Mind \& Society (2015) 1-13.

[112] L. Bargigli, G. di Iasio, L. Infante, F. Lillo, F. Pierobon, The multiplex structure of interbank networks, Quantitative Finance 15 (4) (2015) 673-691. doi : 10.1080/14697688.2014. 968356.

URL http: //dx .doi .org/10.1080/14697688.2014.968356

[113] J. Donges, H. Schultz, N. Marwan, Y. Zou, J. Kurths, Investigating the topology of interacting networks, The European Physical Journal B 84 (4) (2011) 635-651. doi: 10. $1140 /$ epjb/e2011-10795-8.

URL http://dx . doi .org/10.1140/epjb/e2011-10795-8 